\title{
Fungicidal Properties of the Medium from SCOBY Microorganism Cultivation in Saturated Wood against Coniophora puteana Fungus
}

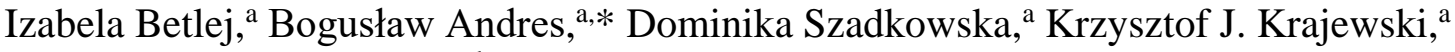 \\ and Aleksandra Ościłowska ${ }^{b}$
}

\begin{abstract}
Effects were assessed for the post-culture liquid medium originating from the cultivation of microorganisms that are present in the ecosystem called symbiotic consortium bacteria and yeast (SCOBY). The effectiveness of protecting Scotch pine wood samples against decomposition caused by the fungus Coniophora puteana was evaluated. The obtained results confirmed that impregnation of wood with post-culture medium reduces the possibility of decomposition of this wood by the test fungus. The potential biocidal effect of the post-culture medium from SCOBY was attributed to the presence the substances of a potential fungicidal nature, which were synthesized in the culture medium during metabolic processes occurring in the culture. The obtained results encourage further studies on the potential use of metabolites obtained from SCOBY breeding to protect wood against biodegradation.
\end{abstract}

Keywords: Fungicides; Post-culture liquid medium; SCOBY microorganisms

Contact information a: Institute of Wood Science and Furniture, Warsaw University of Life ScienceSGGW, 159 Nowoursynowska St., 02-776 Warsaw, Poland; b: Faculty of Wood Technology - Warsaw University of Life Science - SGGW, 159 Nowoursynowska St., 02-776 Warsaw, Poland;

*Corresponding author: boguslaw_andres@sggw.edu.pl

\section{INTRODUCTION}

The search for new biocides for effectively protecting wood, on the one hand, and on the other hand, safety for human health and the environment, are the consequences of legal regulations introduced in European Union countries. The implementation of the provisions of Directive 98/8/EC of the European parliament resulted in the withdrawal of many effective biocides from the market (Directive EC, 1998). Because of their high toxicity, these biocides did not meet the standards for acceptable risk and exposure assessment. The overriding legal document regulating the trade in biocidal products currently in force on the European Union market is the regulation of the European parliament and of the Council No. 528/2012 (EC EU Regulation 2012) concerning the availability on the market and use of biocidal products. Despite the restrictive regulations on the marketing of biocides, the possibility for the research and development work on new biocidal substances and products is still on the rise. Bearing in mind the applicable law, it is worth undertaking research on new ways to protect wood using substances that do not carry any toxicological and ecotoxicological risks. Examples of this type of substance include essential oils, alkaloids, or natural resins obtained from plants or substances produced by microorganisms.

Attempts to use microorganisms or their metabolites to protect wood against decay caused by fungi have been the subject of research by many authors (Highley and Ricard 
1988; Kreber and Morrell 1992; Moita et al. 2005; Susi et al. 2011; Sajitha et al. 2018). Sajitha et al. (2016) isolated and characterized lycopeptides with fungicidal properties, synthesized by the bacterium Bacillus subtilis B1, protecting rubber wood against colonization of the fungus Lasiodiplodia theobromae. Successful attempts to use Bacillus subtilis B1 as a biocontrol agent before fungal growth Ceratocystis sp., Fusarium sp., Aspergillus sp., and Penicillium sp. were conducted by Sajitha et al. (2018). Vicente et al. (2013) found that the active substances produced by Bacillus amyloliquefaciens inhibited the growth of 15 species of fungi. Attempts have been made to use the Pseudomonas putida bacteria, actinomycetes Streptomyces sp., Saccharomyces cerevisiae yeast, and mold fungi to protect ponderosa pine against color changes caused by phytopathogens (Kreber and Morrell 1992). The fungicidal properties of metabolites produced by Streptomyces exfoliatus MT9 were also evaluated (Sharma et al. 2016). The authors of the study showed that the ability of actinomycetes to inhibit the growth of fungi is associated with the synthesis of enzymes such as chitinase and $\beta-1,3$ glucanase, which cause cell wall lysis.

An interesting solution to protect oriented strand boards (OSB) against the development of mold and basidiomycetes was proposed by Yang et al. (2007). The authors of the study used extracts from Phaeotheca dimorphospora fungus cultures for saturating the OSB samples. After 8 weeks of incubation with mold fungi and fungi causing deep decomposition of wood on OSB boards, it was found that Phaeotheca dimorphospora extracts protect samples of wood-based materials against fungal colonization. The studies of the antagonistic ability of Trichoderma viride and Gliocladium virens in relation to fungi that cause deep wood decay were the subject of research by Highley and Ricard (1988). Benitez et al. (2004) showed that the biocontrol properties of Trichoderma species before the development of phytopathogens in wood resulted not only from the ability of fungi to produce metabolites of antibiotic importance, but also from purely environmental mechanisms, such as competition for nutrients and space. Similar observations were made by Smolińska et al. (2014).

An interesting study in the protection of wood is an attempt to assess the fungicidal properties of metabolites produced by microorganisms belonging to the kombucha ecosystem. The kombucha ecosystem consists of bacteria, mainly belonging to Komagataeibacter, with the dominant species of Acetobacter xylinum, Aerobacter, Achromobacter, Agrobacterium, Pseudomonas, and Sarcina, Zooglea i Rhizobium. Mayser et al. (1995) identified yeast-like fungi belonging to Brettanomyces, Zygosaccharomyces, Saccharomyces, and Candida in fermented kombucha drinks. During the fermentation process, these microorganisms produce a number of compounds with antimicrobial properties (Ivanišová et al. 2020). Sreeramulu et al. (2001) identified numerous substances in fermented kombucha drink that exhibit potential bactericidal activity. The killing properties of fermented kombucha drinks against dangerous pathogens, such as Staphylococcus aureus, Shigella sonnei, Escherichia coli, Aeromonas hydrophila, Yersinia enterocolitica, Pseudomonas aeruginosa, Enterobacter cloacae, Staphylococcus epidermis, Campylobacter jejuni, Salmonella enteritidis, Salmonella typhimurium, Bacillus cereus, Helicobacter pylori, and Listeria monocytogenes have been studied by Sreeramulu et al. (2000). The fungicidal properties of the post-culture medium from $A$. xylinum bacteria were observed by Betlej et al. (2020). Researchers found that small amounts of bacterial post-culture medium are sufficient to completely inhibit the growth of fungi that cause deep wood decay. The ability of kombucha to inhibit the growth of Aspergillus flavus and Candida albicans was also observed by Yuniarto et al. (2016). The biocidal properties of the kombucha culture fluid are related to the presence of numerous 
metabolites produced during the culture. Identification and characterization of substances with potential biocidal activity is crucial in the context of planning the possibility of using kombucha in the formulation of wood preservatives.

This study attempts to assess the effectiveness of wood protection with post-culture liquid medium, derived from the cultivation of SCOBY microorganisms, rich in numerous substances with a potential fungicidal effect, against the Coniophora puteana fungus causing brown decomposition of wood. Identification of substances with a potential fungicidal effect was carried out using the GCMS method. The use of gas chromatography allows the detection of volatile substances with low molecular weight. According to numerous scientific studies conducted at the symbiotic consortium bacterial and yeast SCOBY, it is precisely the numerous compounds and low-molecular substances produced in fermentation processes that often exhibit strong antimicrobial properties (Tu et al. 2019).

\section{EXPERIMENTAL}

\section{Materials}

The post-culture liquid derived from the cultivation of SCOBY (Organic farm Wolanin, Szczawnik, Poland) microorganisms was used as the testing sample. The microorganisms were homogenized, and then $1 \mathrm{~mL}$ of the homogenate was added to 100 $\mathrm{mL}$ of the medium containing sucrose, peptone, and yeast extract to produce starter cultures. 10 days after inoculation, the entire starter culture (cellulose with post-culture fluid) was added to $1000 \mathrm{~mL}$ of substrate, which was used to impregnate the wood. The weight of cellulose produced in the starter culture was $21.058 \mathrm{~g}$. SCOBY microorganisms were grown on liquid medium containing 10\% sucrose (Polski cukier, Toruń, Poland), $0.3 \%$ peptone (Biomaxima, Lublin, Poland), and $0.5 \%$ yeast extract (Biomaxima, Lublin, Poland). The microorganisms were cultured for 30 days in the liquid medium in an incubator (Selecta, Barcelona, Spain) in the dark. The temperature and humidity conditions of the culture were: $26 \pm 2{ }^{\circ} \mathrm{C}$ and $66 \pm 2 \%$. After the assumed cultivation time, the cellulose produced on its surface was removed from the medium, and the post-culture liquid medium was sterilized by passing it through syringe filters with a pore diameter of $0.22 \mu \mathrm{m}$ to eliminate the microorganisms contained in it. The post-culture medium, prepared in this way was used for saturating the wood samples. Pine samples (Pinus sylvestris L.) with dimensions according to GOST 16712-71 (1971) standard were used. Wood samples containing only sapwood, without knots or other defects, with an average density of 0.50 $\mathrm{g} / \mathrm{cm}^{3}$, were taken randomly from a previously prepared batch of samples. The average absolute humidity of sample was $7 \%$.

The evaluation of the fungicidal effect of the post-culture medium from SCOBY microorganisms was conducted against the fungus Coniophora puteana (Schumach.) P. Karst. strain EB 97. Basal fungal cultures were grown on a maltose-agar medium containing 2.5\% maltose extract (Biomaxima, Lublin, Poland) and 2.5\% agar (Difco, Franklink Lakes, NJ, USA). The strains of fungi used in the study were obtained from the collection of the Department of Wood Science and Wood Protection, Warsaw University of Life Science - SGGW in Warsaw, Poland. 


\section{Methods}

Assessment of fungicidal properties

The assessment of the fungicidal properties of the post-culture medium from the cultivation of SCOBY microorganisms was performed based on the requirements of Polish Standard, introducing European Standard PN-EN 113 (2000).

Before impregnation, the wood samples were dried at a temperature of $103 \pm 2{ }^{\circ} \mathrm{C}$ for $24 \mathrm{~h}$. After this time, they were weighed to determine the initial dry weight. Saturation of the samples was performed in a vacuum oven (ShelLab, Cornelius, OR, USA). The saturation parameters used were as follows: pressure $150 \mathrm{mbar}$, saturation time $45 \mathrm{~min}$. After saturation, the wood samples were dried, weighed with an accuracy of $0.01 \mathrm{~g}$, and then dried under the same conditions as before saturation. Wood samples were sterilized in a steam autoclave (Spółdzielnia Mechaników SMS, Warsaw, Poland). The wood sample retention $\left(\mathrm{kg} / \mathrm{m}^{3}\right)$ was calculated by following Eq. 1,

$$
R_{\mathrm{c}}=m_{\mathrm{c}} / V
$$

where $R_{\mathrm{c}}$ denotes the liquid retention $\left(\mathrm{kg} / \mathrm{m}^{3}\right), m_{\mathrm{c}}$ represents liquid mass $(\mathrm{kg})$, and $V$ is sample volume $\left(\mathrm{m}^{3}\right)$

The evaluation of the fungicidal properties of the culture medium in saturated wood was performed in Petri dishes onto which the $C$. puteana mycelium had previously been inoculated. The wood samples were placed on sterile glass pads. Fifty wood samples were used for the tests: 30 test samples were saturated with post-culture medium, 10 control samples were used for contact with the mycelium, and 10 control samples as the so-called control without fungus. This term applies to samples saturated with biocide, the so-called control samples, used to calculate the correction factor (C), i.e. changes in the weight of test samples resulting from reasons other than wood decomposition by test fungi. The fungus was cultivated in a heat incubator (Selecta, Barcelona, Spain) for 8 weeks. The temperature and humidity conditions of the cultivation were $22 \pm 2{ }^{\circ} \mathrm{C}$ and $70 \pm 5 \%$.

\section{Identification of volatile substances of low molecular weight in the post-culture medium by gas chromatography coupled with a mass spectrometer}

The analysis of components was performed using a Shimadzu (Kyoto, Japan) gas chromatograph GC-2010 connected to the GCMS-QP2010 mass spectrometer. A ZB-5MS capillary column with a length of $30 \mathrm{~m}$, a diameter of $0.25 \mathrm{~mm}$, and a deposit of $0.25 \mu \mathrm{m}$ was used. Samples were introduced to the column with the AOC-20i autosampler. The samples were analyzed via a dedicated program - GCMS solution Version 2.72. The peaks were identified with NIST11 and NIST11b libraries.

The gas chromatograph-mass spectrometry (GC-MS) analysis program was as follows: Temperature, $50{ }^{\circ} \mathrm{C}$ maintained for $5 \mathrm{~min}$, increased to $200{ }^{\circ} \mathrm{C}$ at $13{ }^{\circ} \mathrm{C} / \mathrm{min}$ rate, increased later to $300{ }^{\circ} \mathrm{C}$ and maintained for $10 \mathrm{~min}$; a flow rate of $0.8 \mathrm{~mL} / \mathrm{min}$; injection temperature of $250{ }^{\circ} \mathrm{C}$; the detector voltage of $1.1 \mathrm{kV}$; and carrier gas of helium - "Gas helium 5.0" (PGNiG, Warsaw, Poland).

\section{Polarimetric determination of sucrose in the culture medium}

The concentration of sucrose in the post-culture medium was determined by the polarimetric method, using a model P3000 polarimeter (Krüss, Hamburg, Germany). Prior to measurement, the culture medium was purified using syringe filters. Measurement of the specific rotation of the sugar solution was performed in a glass tube with a length of 
$1.0 \mathrm{dm}$. The sucrose content of the SCOBY culture medium was determined using Eq. 2 (Szczeniowski 1983),

$$
S=s \times\left(l_{1} / l_{2}\right)
$$

where $S$ represents tested concentration (\%), $s$ denotes concentration of sucrose in medium at the beginning of the cultivation $(\%), l_{1}$ is the specific rotation capacity of the nutrient solution before cultivation of SCOBY microorganisms $\left({ }^{\circ}\right)$, and $l_{2}$ is the specific rotation capacity of the nutrient solution after the cultivation $\left({ }^{\circ}\right)$.

\section{Statistical analysis}

The results were statistically analyzed by performing an analysis of variance for a single classification using the Snedecor statistic (F statistic). Statistical inference was performed for the significance level $\alpha=0.05$. Then, the means of the individual sample groups were compared using the multiple comparison test (post hoc test). For this purpose, the Tukey test was used. Statistical analyzes were developed using Statistica version 13.3 software (StatSoft Polska, Cracov, Poland).

\section{RESULTS AND DISCUSSION}

The fungicidal effectiveness of the post-culture medium from the cultivation of SCOBY against $C$. puteana was assessed from the average weight loss of wood samples, as presented in Table 1.

Table 1. The Effectiveness of Wood Protection with Post-Culture Medium from SCOBY Cultivation against $C$. puteana

\begin{tabular}{|c|c|c|c|c|c|c|c|c|c|}
\hline \multirow{3}{*}{ Samples } & \multicolumn{3}{|c|}{$\begin{array}{c}\text { Average Wood Mass at MC } \\
=0 \%\end{array}$} & \multicolumn{3}{|c|}{ Loss of Wood Mass } & \multirow{2}{*}{$\begin{array}{c}\text { Average } \\
\text { Corrected } \\
\text { Loss of } \\
\text { Mass }\end{array}$} & \multirow{2}{*}{ SD } & \multirow{2}{*}{ CV } \\
\hline & Initial & $\begin{array}{c}\text { After } \\
\text { Saturation }\end{array}$ & Final & Min. & Average & Max. & & & \\
\hline & (g) & (g) & (g) & (g) & (g) & (g) & $(\%)$ & (g) & $(\%)$ \\
\hline $\begin{array}{c}\text { Test } \\
\text { specimen }\end{array}$ & 4.42 & 4.66 & 4.41 & 0.16 & 0.26 & 0.42 & 4.34 & 0.045 & 17.49 \\
\hline Control & 4.43 & 4.43 & 3.98 & 0.34 & 0.49 & 0.65 & 9.40 & 0.08 & 16.33 \\
\hline $\begin{array}{l}\text { Control } \\
\text { without } \\
\text { fungus }\end{array}$ & 4.41 & 4.41 & 4.34 & 0.06 & 0.07 & 0.08 & & & \\
\hline
\end{tabular}

CV- Coefficient of variation, SD - Standard deviation

The obtained results indicated that fungal activity on test samples caused an average of $4.34 \%$ weight loss. At the same time, it was found that the weight loss in the control sample was less than $10 \%$. Therefore, it should be stated that impregnation of wood with SCOBY post-culture medium has a protective effect against the destructive influence of the fungus, which causes brown decomposition of wood. The analysis of variance made it possible to determine the significant differences between the weight loss of the test and control samples caused by the action of $C$. puteana (factor A) (Table 2). The observed high coefficient of variation indicated a strong differentiation of the feature, which in the case of biological tests involved the use of a biocide of natural origin, could be considered as an acceptable result. The high coefficient of variation may result from the different retentions 
of bioactive substances in the impregnated wood. The sucrose content in the post-culture liquid medium, which was used to saturate the wood, was $2.17 \%$. Therefore, it can be concluded that such a concentration of sugar in the post-culture medium may stimulate the growth of the fungus. However, it should be noted that despite the remains of sucrose in the post-culture medium, the inhibitory effect of the fungus growth on the test samples was visible, compared to the results obtained for the control wood.

Table 2. Analysis of Variance and Division of Sample Groups in Tukey Test

\begin{tabular}{|c|c|c|c|}
\hline Variability & Femp. & p-value & $F_{0.05}$ \\
\hline Factor A & 109.83685 & $4.936 \times 10^{-12}$ & 4.13925 \\
\hline \multicolumn{3}{|c|}{} \\
\hline Samples & Average & Sample Groups According to Tukey Test \\
\hline Control & -0.257 & a \\
\hline Test & -0.486 & b \\
\hline
\end{tabular}

Thus far, there have been no available data in the literature on the effectiveness of protecting softwood or hardwood with SCOBY extracts or microorganisms. Research by Betlej et al. (2020), carried out as a nutrient test, confirm the ability of the liquid from $A$. xylinum culture to inhibit the growth of wood-decaying fungi. The ability of kombucha microorganisms (SCOBY) to synthesize antimicrobial substances that are natural preservatives is established and analyzed in detail in the context of food quality and safety. Battikh et al. (2013) demonstrated the killing properties of fermented kombucha against various Candida species. The bactericidal properties of kombucha tea against numerous groups of bacteria were confirmed by Al-Kalifawi (2014) and Ebrahimi Pure and Ebrahimi Pure (2016). On the basis of the conducted analyzes, nine substances have been identified with a biocidal effect, and three substances that may indirectly affect the growth activity of $C$. puteana. The fungicidal properties of hexadecanoic acid were demonstrated by Bukhari et al. (2020). The fungicidal effectiveness of 5-hydroxymethylfurfural was positively assessed by Elsherbiny et al. (2015). Yuniarto et al. (2016) reports that the factor inhibiting the growth activity of microorganisms is, among others, acetic acid, produced during the fermentation process conducted by kombucha microorganisms.

Table 3. List of Substances Identified in Liquid Kombucha Culture Medium with Potential Importance in Inhibiting the Growth of C. puteana

\begin{tabular}{|c|c|}
\hline Substances with a Fungicidal Effect & Concentration (\%) \\
\hline Hexadecanoic acid, ethyl ester & 1.03 \\
n-Hexadecanoic acid & 0.59 \\
2H-Pyran-2,6(3H)-dione & 26.63 \\
Acetic acid, hydroxy-, ethyl ester & 12.76 \\
Benzoic acid & 1.57 \\
D-Gluconic acid, ס-lactone & 0.60 \\
5-Hydroxymethylfurfural & 25.83 \\
D-Galactonic acid, y-lactone & 0.60 \\
2-Pentenoic acid, 4-methyl- & 2.86 \\
\hline Substances with an Indirect Fungicidal Effect & Concentration (\%) \\
\hline 5-Acetoxymethyl-2-furaldehyde & 0.77 \\
Butanedioic acid, diethyl ester & 1.77 \\
Manganese, pentacarbonylethyl-, (oc-6-21)- & 3.58 \\
\hline
\end{tabular}


Biocidal properties can also be attributed to substances such as lactic acid, glucuronic acid, ethanol, and usnic acid (Blanc 1996; Cardoso et al. 2020), produced as products of SCOBY metabolism. Polyphenol fractions, such as catechin and isorhamnetin, produced by SCOBY influence on the physiological activity of microorganisms (Bhattacharya et al. 2020) by neutralizing bacterial toxins or inhibiting biofilm formation (Cardoso et al. 2018).

The method used allowed the identification of volatile, low-molecular compounds of potential fungicidal importance. However, it is not excluded that the composition of the medium derived from the cultivation of SCOBY microorganisms may contain many other substances that have not been identified due to the limitations of this method. There is a probability that the composition of the post-culture medium may contain numerous enzymes and bacteriocins that exhibit fungicidal properties (Bhattacharya et al. 2020, Tu et al. 2019).

\section{CONCLUSIONS}

1. Post-culture liquid medium, obtained from the cultivation of kombucha microorganisms, inhibits the decomposition of wood caused by $C$. puteana.

2. Nine substances with potential antimicrobial properties were found in the kombucha culture medium.

\section{ACKNOWLEDGMENTS}

The financial support from Warsaw University of Life Sciences - SGGW is thankfully acknowledged.

\section{REFERENCES CITED}

Al-Kalifawi, E. J. (2014). "Study the antimicrobial effect of kombucha tea on bacteria isolated from diabetic foot ulcler," International Journal for Sciences and Technology 9(1), 49-56. DOI: 10.12816/0010111

Battikh, H., Chaieb, K., Bakhrouf, A., and Ammar, E. (2013). "Antibacterial and antifungal activities of black and green kombucha teas," Journal of Food Biochemistry 37(2), 231-236. DOI: 10.1111/j.1745-4514.2011.00629.x

Bhattacharya, D., Sinha, R., Mukherjee, P., Howlader, D. R., Nag, D., Sarkar, S., Koley, H., Withey, J. H., and Gachhui, R. (2020). "Anti-virulence activity of polyphenolic fraction isolated from Kombucha against Vibrio cholerae," Microbial Phathogenesis 140, Article ID 103927. DOI: 10.1016/j.micpath.2019.103927

Benitez, T., Rincón, A. M., Limón, M. C., and Codón, A. C. (2004). "Biocontrol mechanisms of Trichoderma strains," International Microbiology 7(4), 249-260. DOI: 10.2436/IM.V7I4.9480

Betlej, I., Andres, B., and Krajewski, K. J. (2020). "Evaluation of fungicidal effects of post-culture medium of selected mold fungi and bacteria in relation to Basidiomycetes 
fungi causing wood destruction," BioResources 15(2), 2471-2482. DOI:

10.15376/biores.15.2.2471-2482

Blanc, P. J. (1996). "Characterization of the tea fungus metabolites," Biotechnology Letters 18(2), 139-142. DOI: 10.1007/BF00128667

Bukhari, S. A., Salman, M., Numan, M., Javed, M. R., Zubair, M., and Mustafa, G. (2020). "Characterization of antifungal metabolites produced by Lactobacillus plantarum and Lactobacillus coryniformis isolated from rice rinsed water," Molecular Biology Reports 47(3), 1871-1881. DOI: 10.1007/s11033-020-05281-1

Cardoso, O., Neto, R. O., dos Santos D'Almeida, C. T., do Nasimento, T. P., Pressete, C. G., Azevedo, L., Martino, H. S. D., Cameron, L. C., Ferreira, M. S. L., and de Barros, F. A. R. (2020). "Kombuchas from green and black teas have different phenolic profile, which impacts their antioxidant capacities, antibacterial and antiproliferative activities," Food Research International 128, Article ID 108782. DOI: 10.1016/j.foodres.2019.108782

Cardoso, O., Donato, M. M., Luxo, C., Almeida, N., Liberal, J., Figueirinha, A., and Batista, M. T. (2018). "Anti-helicobacter pylori potential of Agrimonia eupatoria L. and Fragaria vesca," Journal of Functional Foods 44(5), 299-303. DOI: 10.1016/j.jff.2018.03.027

Directive 98/8/EC (1998). “The Biocidal Products Directive, European Parliament and the Council of 16 February 1998, concerning the placing of biocidal products on the market," European Union, Brussels, Belgium.

Ebrahimi Pure, A., and Ebrahimi Pure, M. (2016). "Antioxidant, antibacterialand color analysis of garlic fermented in kombucha and red grape vinegar," Applied Food Biotechnology 3(4), 246-252. DOI: 10.22037/afb.v3i4.12312

Elsherbiny, E. A., Saad, A. S., Zaghloud, M. G., and El-Sheshtawi, M. A. (2015). "Efficiency assessment of the antifungal metabolites from Sclerotium cepivorum against onion white rot disease," European Journal of Plant Pathology 142(4), 843854. DOI: 10.1007/s10658-015-0656-1

European Commission (EC) Regulation (EU) No 528/2012 (2012). "Regulation (EU) No 528/2012 European Parliament and of the Council of 22 May concerning the making available on the market and use of biocidal products," European Union, Brussels, Belgium.

GOST 16712-71 (1971). "Wood preservatives. Method of testing for toxicity," State Committee of Standards of the Council of Ministers, Moscow, Russia.

Highley, T. L., and Ricard, J. (1988). "Antagonism of Trichoderma spp. and Gliocladium virens against wood decay fungi," Material und Organismen 23(3), 157-169.

Ivanišová, E., Meňhartová, K., Terentjeva, M., Harangozo, L., Kántor, A., and Kačániová, M. (2020). "The evaluation of chemical, antioxidant, antimicrobial and sensory properties of kombucha tea beverage," Journal of Food Science and Technology -Mysore 57(5), 1840-1846. DOI: 10.1007/s13197-019-04217-3

Kreber, B., and Morrell, J. J. (1992). "Ability of selected bacterial and fungal bioprotecants to limit fungal stain in ponderosa pine wood," Wood and Fiber Science 25(1), 23-34.

Mayser, P., Fromme, S., Leitzmann, C., and Gründer, K. (1995). "The yeast spectrum of the tea fungus Kombucha," Mycoses 38(7-8), 289-295. DOI: 10.1111/j.14390507.1995.tb00410.x

Moita, C., Feio, S. S., Nunes, L., Curto, M. J. M., and Roseiro, J. C. (2005). "Optimisation of physical factors on the production active metabolites by Bacillus 
subtilis 355 against wood surface contaminant fungi," International Biodeterioration and Biodegradation 55(4), 261-269. DOI: 10.1016/j.ibiod.2005.02.003

PN - EN 113 (2000). "Wood preservatives - Test method for determining the protective effectiveness against wood destroying basidiomycetes - Determination of the toxic values," Polish Committee for Standardization, Warsaw, Poland.

Sajitha, K. L., Dev, S. A., and Florence, E. J. M. (2016). "Identification and characterization of lipopeptides from Bacillus subtilis B1 against sapstain fungus of rubberwood through MALDI-TOF-MS and RT-PCR," Current Microbiology 73(1), 46-53. DOI: 10.1007/s00284-016-1025-9

Sajitha, K. L., Dev, S. A., and Florence, E. J. M. (2018). "Biocontrol potential of Bacillus subtilis B1 against sapstain fungus in rubber wood," European Journal of Plant Pathology 150(1), 237-244. DOI: 10.1007/s10658-017-1272-z

Sharma, P., Choudhary, B., Nagpure, A., and Gupta, R. K. (2016). “Antifungal potential of actinomycete isolate Streptomyces exfoliatus MT9 against wood-rotting fungi," Journal of Environmental Biology 37(6), 1231-1237. DOI:

10.22438/jeb/37/6/mrn.1000

Smolińska, U., Kowalska, B., Kowalczyk, W. and Szczech M. (2014). "The use of agroindustrial wastes as carriers of Trichoderma fungi in the parsley cultivation," Scientia Horticulturae 179(15), 1-8. DOI: 10.1016/j.scienta.2014.08.023

Sreeramulu, G., Zhu, Y., and Knol, W. (2000). "Kombucha fermentation and its antimicrobial activity," Journal of Agricultural and Food Chemistry 48(6), 25892594. DOI: 10.1021/jf991333m

Sreeramulu, G., Zhu, Y., and Knol, W. (2001). "Characterization of antimicrobial activity in kombucha fermentation," Acta Biotechnologica 21(1), 49-56. DOI: 10.1002/15213846(200102)21:1<49::AID-ABIO49>3.0.CO;2-G

Susi, P., Aktuganov, G., Himanen, J., and Korpela, T. (2011). "Biological control of wood decay against fungal infection,” Journal of Environmental Management 92(7), 1681-1689. DOI: 10.1016/j.jenvman.2011.03.004

Szczeniowski, S. (1983). Experimental Physics, Volume 4, Polish Scientific Publishing House PWN, Warszawa, Poland.

Tu, Ch., Tang, S., Azi, F., Hu, W. and Dong, M. (2019). "Use of kombucha consortium to transform say whey into a novel functional beverage," Journal of Functional Food 52(1), 81-89. DOI:10.1016/j.jff.2018.10.024

Vicente, H., Roseiro, J. C., Arteiro, J. M., Neves, J., and Caldeira, A. T. (2013). "Prediction of bioactive compound activity against wood contaminant fungi using artificial neural networks," Canadian Journal of Forest Research 43(1), 1-8. DOI: $10.1139 /$ cjfr-2013-0142

Yang, D.-Q., Wan, H., Wang, X.-M., and Liu, Z.-M. (2007). "Use of fungal metabolites to protect wood-based panels against mould infection," BioControl 52(3), 427-436. DOI: $10.1007 / \mathrm{s} 10526-006-9022-8$

Yuniarto, A., Anggadiredja, K., and Aqidah, R. A. N. (2016). "Antifungal activity of Kombucha tea against human pathogenic fungi," Asian Journal of Pharmaceutical and Clinical Research 9(5), 253-255. DOI: 10.22159/ajpcr.2016.v9i5.13432

Article submitted: October 22, 2020; Peer review completed: October 25, 2020; Revised version received and accepted: December 14, 2020; Published: December 23, 2020. DOI: 10.15376/biores.16.1.1287-1295 